\title{
ASSESSMENT OF GENETIC RELATIONSHIPS AMONG CULTIVATED AND WILD PISTACHIOS (PISTACIA VERA L.) USING MOLECULAR MARKERS
}

\author{
Mohammad Aref POURIAN ${ }^{1}$, Davood BAKHSHI ${ }^{*}$, Ali AALAMI ${ }^{1}$, Hossein HOKMABADI $^{2}$ \\ ${ }^{1}$ University of Guilan, Iran \\ ${ }^{2}$ Iranian Pistachio Research Institute, Iran
}

Received: December 2018; Accepted: June 2019

\begin{abstract}
Iran is one of the main diversity centers and origins of pistachios in the world. Pistachio cultivation spread first within the ancient Persian Empire and then moved gradually westward. Knowledge of the genetic relationships among wild and cultivated varieties of pistachio is important for the efficient utilization of the available germplasm resources. Three molecular marker strategies, namely, inter-simple sequence repeat (ISSR), inter-retrotransposon amplified polymorphism (IRAP), and retrotransposon microsatellite amplified polymorphism (REMAP), were used to study the genetic relationships among 35 pistachio accessions including 15 wild-type genotypes of Pistacia vera and 20 important cultivars from Iran. According to the results, high levels of polymorphism were observed for all three marker systems. REMAP and IRAP techniques had the higher mean values of genetic relationships parameters than ISSR technique. The results from this study showed that the 5'LTR2, Sukkula, Sukkula + UBC855, and 5'LTR2 + UBC811 primers were the most informative and could be used to evaluate the genetic relationships of pistachios accessions. Cluster analysis using the unweighted pair group method with arithmetic mean (UPGMA) properly separated the accessions and divided them into four main groups. The presence of most cultivated genotypes in a group indicates genetic erosion of cultivated pistachio in Iran. Wild-type genotypes of $P$. vera are located in different clusters indicating the high diversity of the genotypes. The results provide useful genetic information about wild pistachios in northeastern of Iran and indicate that the use of wild pistachios in breeding programs could be useful for generating new genotypes with interesting characters.
\end{abstract}

Keywords: genetic relationship, molecular markers, microsatellite, Pistacia, retrotransposon

\section{INTRODUCTION}

The Anacardiaceae family consists of about 82 genera and more than 700 species distributed primarily in tropical and subtropical areas of the world (Pell 2004). This family is well-known for its cultivated edible fruits and seeds such as pistachios (Pistacia vera L.), mangos (Mangifera indica L.), and cashews (Anacardium occidentale L.) (Pell 2004). P. vera L. is the only cultivated species in the genus Pistacia. The genus Pistacia contains only 11 species, of which $P$. vera is the most economically important ones (Zohary 1952). The pistachio is believed to have originated in the arid zones of Central Asia (Crane 1979; Parfitt \& Badenes 1997); it has been cultivated for 3,000-4,000 years in Iran and was introduced into Mediterranean
Europe by Romans at the beginning of the Christian era (Crane 1979). Iran has one of the richest genetic reserves and is one of the largest producers of pistachio in the world. Nevertheless, pistachio cultivation in Iran is facing some difficult challenges and these problems will be further increased in the future. The fundamental problems facing the pistachio cultivation in Iran include (1) vulnerability and genetic erosion because of the usage of improved varieties and (2) climate change and growing water crisis (Michel 2017). Wild genotypes are very rich gene source and may have genes for diseases and pests resistance and also tolerance to drought stress, cold, and salinity, which can be useful for breeding programs as well as for the introduction of new important cultivated pistachios in the future (He et al. 2015). Knowledge of the genetic 
relationships of plant genetic resources is fundamental for breeding programs (Dempewolf et al. 2017). Genetic markers can be used in plant breeding programs for germplasm evaluation and assessment of genetic diversity (Yang et al. 2015). In recent years, studies have been conducted using molecular markers to investigate the diversity and genetic relationships of pistachios, including simple sequence repeat - SSR (Arabnezhad et al. 2011; Ziya Motalebipour et al. 2016), randomly amplified polymorphic DNA RAPD (Hormaza et al. 1998), start codon targeted SCoT and inter-retrotransposon amplified polymorphism - IRAP (Sorkheh et al. 2017), and amplified fragment length polymorphism - AFLP (Shanjani et al. 2009). Inter-simple sequence repeats (ISSRs) technique is one of the polymerase chain reaction (PCR) based method, which involves the amplification of DNA segment present at an amplifiable distance between two identical microsatellite repeat regions oriented in opposite direction (Reddy et al. 2002). ISSRs are quick and easy assay, and there is no need of prior knowledge of DNA sequences (Kar et al. 2005). IRAP is a retrotransposon-type marker that is based on the available retrotransposon sequence information and have been developed for plants (Kalendar et al. 1999). IRAP method can be carried out with a single primer matching with either $5^{\prime}$ or $3^{\prime}$ end of the long terminal repeat (LTR) but oriented away from the LTR itself, or with two LTR primers (Kalendar et al. 2011). Retrotransposon microsatellite amplified polymorphism (REMAP) method is similar to IRAP, but one of the two primers matches a microsatellite motif so that the DNA sequences between the LTRs of a retrotransposon and adjacent microsatellites (SSRs) are amplified using appropriate primers (Kalendar et al. 2011).

The natural habitats of pistachio are located in northeastern Iran. In spite of much attention paid to this important crop plant in Iran, no data on the genetic structure of wild pistachios and their relationships with cultivated genotypes in the natural habitat of Maraveh Tappeh at northeast country are available. Owing to the importance of pistachio and the necessity of using the genetic potential of this plant for breeding purposes, the present study aimed to explore the genetic relationships among cultivated and wild pistachios (Pistacia vera L.) based on the ISSR, IRAP, and REMAP techniques.

\section{MATERIALS AND METHODS}

\section{Plant material}

Thirty-five genotypes of $P$. vera have been used. Accession consisted of 20 cultivated genotypes and 15 wild genotypes (Table 1). Twenty different cultivated genotypes of pistachio obtained from diverse geographical areas maintained at the experimental orchards located at the Buin Zahra in Qazvin, Iran. Fifteen genotypes were selected as representatives of the wild pistachio forests in northeastern Iran. This pistachio habitat is located in the Maraveh Tappeh County, Golestan Province in the geographical coordinates of the region $55^{\circ} 14^{\prime} \mathrm{E}$ to $56^{\circ} 23^{\prime} \mathrm{E}$ longitude and $37^{\circ} 54^{\prime} \mathrm{N}$ to $38^{\circ} 15^{\prime} \mathrm{N}$ latitude with an area of approximately 5,200 ha (Fig. 1). Young leaves of cultivated and wild pistachios were collected and washed in water, frozen in liquid nitrogen and kept at $-80{ }^{\circ} \mathrm{C}$ until DNA extraction.

\section{DNA extraction}

Genomic DNA of samples was extracted from fresh pistachio leaves by a modified cetrimonium bromide (CTAB) method following the procedure described by Hormaza et al. (1998). The quality of DNA samples was determined using $1 \%$ agarose gel electrophoresis in tris-borate-EDTA (TBE) buffer. DNA concentration was quantified using Nano Drop-1000 spectrophotometer (NanoDrop Technologies, USA); diluted to $50 \mathrm{ng} \mu \mathrm{l}^{-1}$ for use in ISSR, IRAP, and REMAP analyses, and stored at $-20^{\circ} \mathrm{C}$.

\section{PCR reaction conditions and program}

Optimal reaction conditions suitable for ISSR, IRAP, and REMAP for the analysis of the pistachio were established. The reaction mixture of $10 \mu \mathrm{l}$ comprising $20 \mathrm{ng}$ of template DNA, $0.4 \mu \mathrm{l}$ of $50 \mathrm{mM} \mathrm{MgCl} 2,0.4 \mu 1$ of $10 \mathrm{mM}$ deoxynucleotides (dNTPs), $1 \mu 1$ of $10 \times$ PCR buffer, $0.1 \mathrm{U}$ Taq DNA polymerase (CinnaGen), and $1.0 \mu \mathrm{l}$ of $10 \mu \mathrm{M}$ of each primer. PCR amplification was conducted in a thermocycler (Mastercycler gradient, Eppendorf, Germany) using the following program: 1 cycle at $94{ }^{\circ} \mathrm{C}, 4 \mathrm{~min} ; 35$ cycles of $94{ }^{\circ} \mathrm{C}, 40 \mathrm{~s} ; 50-65^{\circ} \mathrm{C}$, $40 \mathrm{~s} ; 72{ }^{\circ} \mathrm{C}, 1 \mathrm{~min} ; 1$ cycle at $72{ }^{\circ} \mathrm{C}, 10 \mathrm{~min} ; 4^{\circ} \mathrm{C}$. The annealing temperature of the PCR program was determined by the primer melting temperatures (Tm) value. 


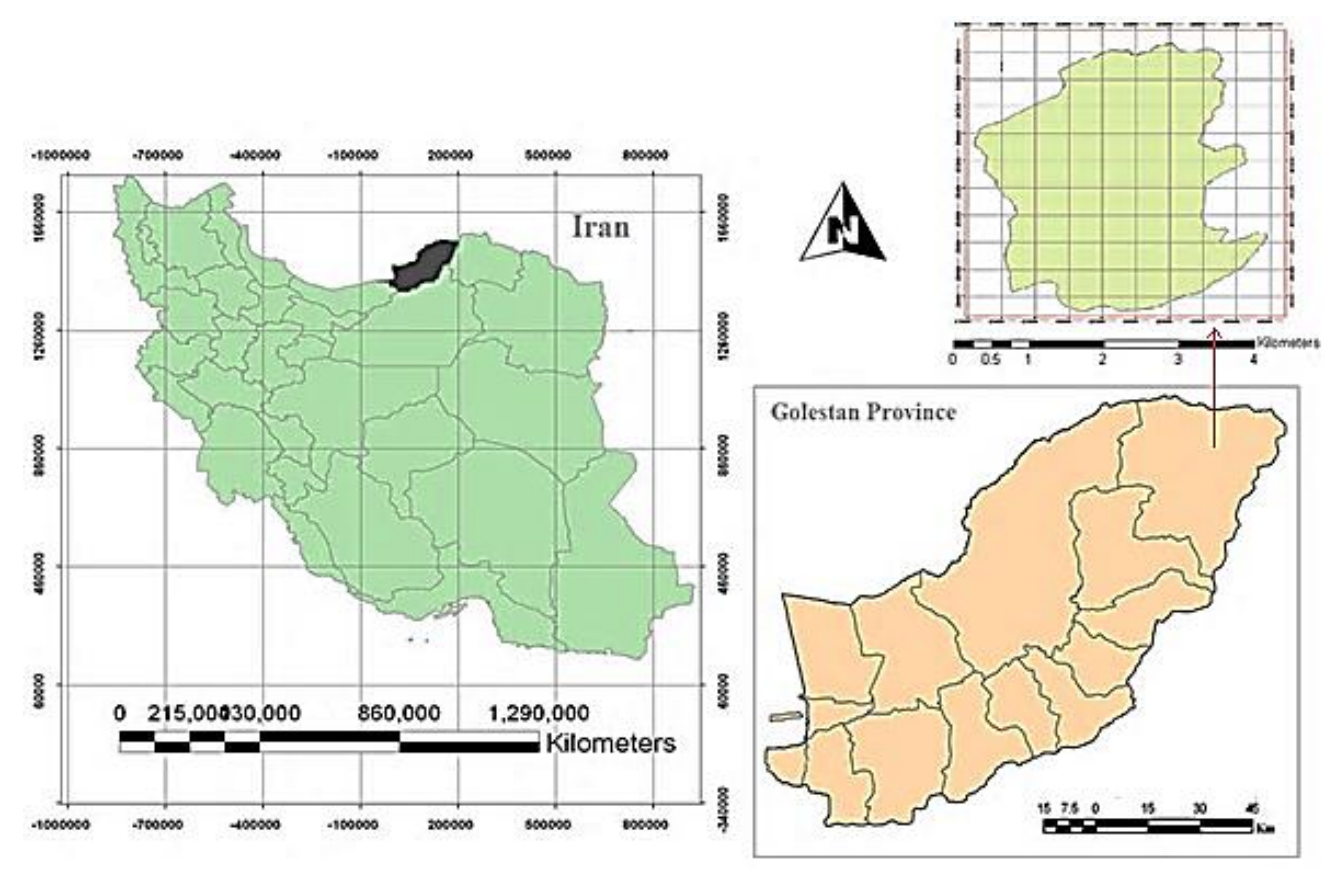

Fig. 1. The location of the habitat pistachio and province and country

Table 1. List of pistachio accessions and their code assessed in this study

\begin{tabular}{llllll}
\hline Code & \multicolumn{1}{c}{ Accession } & Genotype & Code & \multicolumn{1}{c}{ Accession } & Genotype \\
\hline W1 & genotype 1 & wild & G19 & Ahmad Agaei & cultivated \\
W2 & genotype 2 & wild & G20 & Shahpasand 32 & cultivated \\
W3 & genotype 3 & wild & G21 & Pakzadi & cultivated \\
W4 & genotype 4 & wild & G22 & Kaleghochi & cultivated \\
W5 & genotype 5 & wild & G23 & Italiaei riz & cultivated \\
W6 & genotype 6 & wild & G24 & Italiaei Dorosht & cultivated \\
W7 & genotype 7 & wild & G25 & Badami kj & cultivated \\
W8 & genotype 8 & wild & G26 & Razavi & cultivated \\
W9 & genotype 9 & wild & G27 & Momtaz & cultivated \\
W10 & genotype 10 & wild & G28 & Qatruyeh & cultivated \\
W11 & genotype 11 & wild & G29 & Nazari & cultivated \\
W12 & genotype 12 & wild & G30 & Abbasali & cultivated \\
W13 & genotype 13 & wild & G31 & Mohseni & cultivated \\
W14 & genotype 14 & wild & G32 & Rezaei & cultivated \\
W15 & genotype 15 & wild & G33 & Kerman & cultivated \\
G16 & Akbari & cultivated & G34 & Badami Nishkalaghi & cultivated \\
G17 & Fandoghi 48 & cultivated & G35 & Ghazvini & cultivated \\
G18 & Sefidkhorasan & cultivated & & & \\
\hline
\end{tabular}

\section{Data analysis}

The ISSR, IRAP, and REMAP products were separated by electrophoresis on $2 \%$ agarose gel for $2-$ $3 \mathrm{~h}$ at $70 \mathrm{~V}$. The fragment patterns were photographed under UV light in a BioDoc Analyze 2.1 (Germany) for further analysis. The Ladder $100 \mathrm{bp}$ plus R-T-U 100-3000 was used as the molecular standard to confirm the presence of the DNA fragments. From the ISSR, IRAP, and REMAP profiles, all clearly detectable polymorphic and monomorphic bands were scored for the analysis and were scored as 1 and 0 based on the presence and absence 
of the band, respectively. A binary data matrix (1/0) was set up according to whether the bands existed or not. Polymorphic information content (PIC), effective multiplex ratio (EMR), and marker index (MI) were calculated as follows: $\mathrm{PIC}=1-$ $\sum_{i=1}^{n} \mathrm{P}_{i}^{2}$, where: $n$ is the total number of alleles detected for a given marker locus and $\mathrm{P}_{i}$ is the frequency of the $\mathrm{i}$-th allele (Smith et al. 1997); EMR = $n_{p} \times \beta$, where $n_{p}$ is the total of polymorphic bands, and $\beta$ is the ratio of polymorphic bands to total bands and MI $=$ PIC $\times$ EMI (Powell et al. 1996).

The effective number of alleles (Ne), Nei's gene diversity (h), and Shannon's information index (l) were computed using POPGENE32 software (Yeh et al. 1999). Jaccard's similarity coefficients were calculated using the numerical taxonomy multivariate analysis system NTSYSpc version 2.02 (Rohlf 2009). The resulting matrices were used for the construction of dendrogram using the unweighted pair group method with arithmetic mean (UPGMA). To measure the goodness of fit between dendrogram and the similarity matrix on which it was based, the cophenetic correlation coefficient with 1,000 permutations was calculated. Principal coordinate analysis (PCoA) was performed using Past 3.20 on a similarity matrix.

\section{RESULTS}

\section{Level of polymorphism and discriminating capacity}

Thirty-five pistachio genotypes were uniquely identified by ISSR, IRAP, and REMAP markers in this study (Table 2). The results of three molecular markers used for the evaluation of genetic relationships among Iranian pistachios are summarized in Table 3. In the case of ISSR analysis, 6 ISSR primers were chosen for PCR amplification that generated 50 total and 41 polymorphic bands with a polymorphism rate of $82.83 \%$ among studied pistachio genotypes. The total number of amplified fragments varied from 7 to 10 across the accessions (Table 3). Seven selected IRAP primers produced a total of 57 bands, of which 49 bands were polymorphic (82.34\%) in all 35 accessions. 5'LTR2 detected 15 bands and Sukkula detected 11 bands, and the number of polymorphic bands varied from 3 (TOS2 and WLTR2105) to 15 (5'LTR2) with an average of 8.4 per primer (Table 3). A total of 40 primer combinations were tested for REMAP amplification but only 5 pairs of primers (Table 2) produced unambiguous polymorphic bands and were used to amplify the DNA samples from 35 pistachio genotypes. About REMAP, high polymorphism percentage was obtained from 5 primer pairs $(88.69 \%)$ with a total of 40 bands, of which 36 were polymorphic. The number of polymorphic bands per primer varied from 4 (5'LTR2 + UBC840) to 10 (Sukkula + UBC855) with an average of 7.2 (Table 3). Maximum polymorphism (100\%) was observed with primers 5'LTR2, 5'LTR2 + UBC811, and Sukkula + UBC855, whereas the least level of polymorphism (75\%) was observed with primer TOS2 and WLTR2105 (Table 3).

Comparison of informativeness obtained with ISSR, IRAP, and REMAP markers

Comparison of the main aspects of the performance of the examined three marker systems including PIC, MI, and EMR are presented in Table 3. In our comparison of the molecular markers assays, the PIC values were higher in the REMAP than in the IRAP and ISSR techniques. The mean of PIC values for REMAP, IRAP, and ISSR markers were 0.32, 0.31 , and 0.23 , respectively. PIC values for all the primers used ranged from 0.23 (TOS2) to 0.40 (5'LTR2 and 5'LTR2 + UBC811) (Table 3). In the present study, EMR and MI were relatively higher for IRAP than for REMAP and ISSR markers. The mean of EMR and MI values for IRAP marker were 8.74 and 3.18, respectively (Table 3). For the REMAP markers, the effective number of alleles per locus $(\mathrm{Ne})$ was 1.68 , whereas for IRAP and ISSR, these values were slightly lower (1.67 and 1.63, respectively). The highest assay Nei's gene diversity $(\mathrm{H})$ and Shannon's information index (I) values were observed for $\operatorname{REMAP}(0.37$ and 0.53 , respectively) and the lowest was for ISSR ( 0.34 and 0.49 , respectively). The values for IRAP markers were intermediate between those of REMAP and ISSR (Table 3). The results showed that the UBC811, UBC071, 5'LTR2, Sukkula, 5'LTR2 + UBC811, and Sukkula + UBC855 primers had a greater potential among primers used in this study. Also, 5'LTR2, Sukkula, Sukkula + UBC855, and 5'LTR2 + UBC811 primers were the most informative, which could be used to evaluate the genetic relationships of pistachios accessions. 
Table 2. List of the primers used in this study

\begin{tabular}{|c|c|c|c|c|}
\hline Marker type & No. & Primer name & Nucleotide sequence $\left(5^{\prime}-3^{\prime}\right)$ & $\begin{array}{c}\text { Annealing } \\
\text { temperature }\left({ }^{\circ} \mathrm{C}\right)\end{array}$ \\
\hline \multirow{6}{*}{ ISSR } & 1 & UBC071 & GAGGGCGAGG & 52 \\
\hline & 2 & UBC811 & GAGAGAGAGAGAGAGAC & 52 \\
\hline & 3 & UBC812 & GAGAGAGAGAGAGAGAA & 48 \\
\hline & 4 & UBC840 & GAGAGAGAGAGAGAGAYT & 52 \\
\hline & 5 & UBC857 & ACACACACACACACACYG & 53 \\
\hline & 6 & $\mathrm{UBC} 880$ & GGAGAGGAGAGGAGA & 53 \\
\hline \multirow{7}{*}{ IRAP } & 8 & TOS1 & TGTTGGGAATAGTCCCACA & 52 \\
\hline & 11 & TOS2 & TGTTGAATAGTTCCACATT & 48 \\
\hline & 7 & 5'LTR2 & ATCATTCCCTCTAGGGCATAATTC & 56 \\
\hline & 10 & Sukkula & GATAGGGTCGCATCTTGGGCGTGAC & 65 \\
\hline & 12 & WLTR2105 & ACTCCATAGATGGATCTTGGTGA & 45 \\
\hline & 13 & LTR1061 & AGAGGGGAATGTGGGGGTTTCC & 52 \\
\hline & 9 & LTR2116 & CGAACCTGGGTAAAACTTCGTGTC & 58 \\
\hline \multirow{5}{*}{ REMAP } & 14 & 5'LTR2+UBC811 & ATCATTCCCTCTAGGGCATAATTC $+(\mathrm{GA})_{8} \mathrm{C}$ & 55 \\
\hline & 15 & 5'LTR2+UBC840 & ATCATTCCCTCTAGGGCATAATTC $+(\mathrm{GA})_{8} \mathrm{YT}$ & 53 \\
\hline & 16 & Sukkula+UBC071 & GATAGGGTCGCATCTTGGGCGTGAC + GAGGGCGAGG & 55 \\
\hline & 17 & Sukkula+UBC811 & GATAGGGTCGCATCTTGGGCGTGAC + $(\mathrm{GA})_{8} \mathrm{~A}$ & 56 \\
\hline & 18 & Sukkula+UBC855 & GATAGGGTCGCATCTTGGGCGTGAC + (AC) 8 CTT & 55 \\
\hline
\end{tabular}

Table 3. Levels of polymorphism and comparison of informativeness obtained with ISSR, IRAP, and REMAP markers in 35 pistachio genotypes

\begin{tabular}{|c|c|c|c|c|c|c|c|c|c|c|c|}
\hline Marker type & No. & Primer name & TB & $\mathrm{PB}$ & PPB & PIC & EMR & MI & $\mathrm{Ne}$ & $\mathrm{H}$ & $\mathrm{I}$ \\
\hline \multirow{6}{*}{ ISSR } & 1 & UBC071 & 10 & 8 & 80 & 0.32 & 6.40 & 2.06 & 1.67 & 0.35 & 0.50 \\
\hline & 2 & UBC811 & 10 & 9 & 90 & 0.35 & 8.10 & 2.90 & 1.75 & 0.40 & 0.58 \\
\hline & 3 & UBC812 & 7 & 5 & 77.77 & 0.30 & 3.57 & 1.10 & 1.58 & 0.31 & 0.44 \\
\hline & 4 & UBC840 & 7 & 6 & 85.71 & 0.27 & 5.14 & 1.43 & 1.60 & 0.34 & 0.49 \\
\hline & 5 & UBC857 & 7 & 6 & 85.71 & 0.26 & 5.14 & 1.36 & 1.53 & 0.29 & 0.43 \\
\hline & 6 & UBC880 & 9 & 7 & 77.77 & 0.26 & 5.44 & 1.43 & 1.59 & 0.32 & 0.46 \\
\hline \multicolumn{2}{|c|}{ Mean } & & 8.6 & 7 & 82.83 & 0.30 & 5.73 & 1.78 & 1.63 & 0.34 & 0.49 \\
\hline \multirow{7}{*}{ IRAP } & 7 & TOS2 & 4 & 3 & 75 & 0.23 & 2.25 & 0.51 & 1.53 & 0.28 & 0.41 \\
\hline & 8 & TOS1 & 9 & 7 & 77.77 & 0.31 & 5.44 & 1.74 & 1.55 & 0.30 & 0.44 \\
\hline & 9 & 5'LTR2 & 15 & 15 & 100 & 0.40 & 15 & 6.04 & 1.78 & 0.42 & 0.62 \\
\hline & 10 & Sukkula & 11 & 10 & 90.90 & 0.37 & 9.09 & 3.43 & 1.83 & 0.43 & 0.60 \\
\hline & 11 & WLTR2105 & 4 & 3 & 75. & 0.30 & 2.25 & 0.67 & 1.55 & 0.31 & 0.45 \\
\hline & 12 & LTR1061 & 5 & 4 & 80 & 0.27 & 3.20 & 0.87 & 1.67 & 0.35 & 0.51 \\
\hline & 13 & LTR2116 & 9 & 7 & 77.77 & 0.27 & 5.44 & 1.50 & 1.55 & 0.30 & 0.44 \\
\hline \multicolumn{2}{|c|}{ Mean } & & 9.6 & 8.4 & 82.35 & 0.31 & 8.74 & 3.18 & 1.67 & 0.36 & 0.52 \\
\hline \multirow{5}{*}{ REMAP } & 14 & 5'LTR2+UBC811 & 9 & 9 & 100 & 0.40 & 9 & 3.65 & 1.67 & 0.38 & 0.56 \\
\hline & 15 & 5'LTR2+UBC840 & 5 & 4 & 80 & 0.23 & 3.20 & 0.74 & 1.59 & 0.31 & 0.45 \\
\hline & 16 & Sukkula+UBC071 & 9 & 7 & 77.77 & 0.29 & 5.44 & 1.58 & 1.73 & 0.37 & 0.52 \\
\hline & 17 & Sukkula+UBC811 & 7 & 6 & 85.71 & 0.29 & 5.14 & 1.53 & 1.72 & 0.38 & 0.54 \\
\hline & 18 & Sukkula+UBC855 & 10 & 10 & 100 & 0.37 & 10 & 3.79 & 1.67 & 0.38 & 0.56 \\
\hline \multicolumn{2}{|c|}{ Mean } & & 8 & 7.2 & 88.69 & 0.32 & 6.55 & 2.26 & 1.68 & 0.37 & 0.53 \\
\hline \multicolumn{2}{|c|}{ Total } & & 147 & 126 & & & & & & & \\
\hline Total mean & & & 8.01 & 7.00 & 84.62 & 0.31 & 6.07 & 2.02 & 1.66 & 0.36 & 0.52 \\
\hline
\end{tabular}




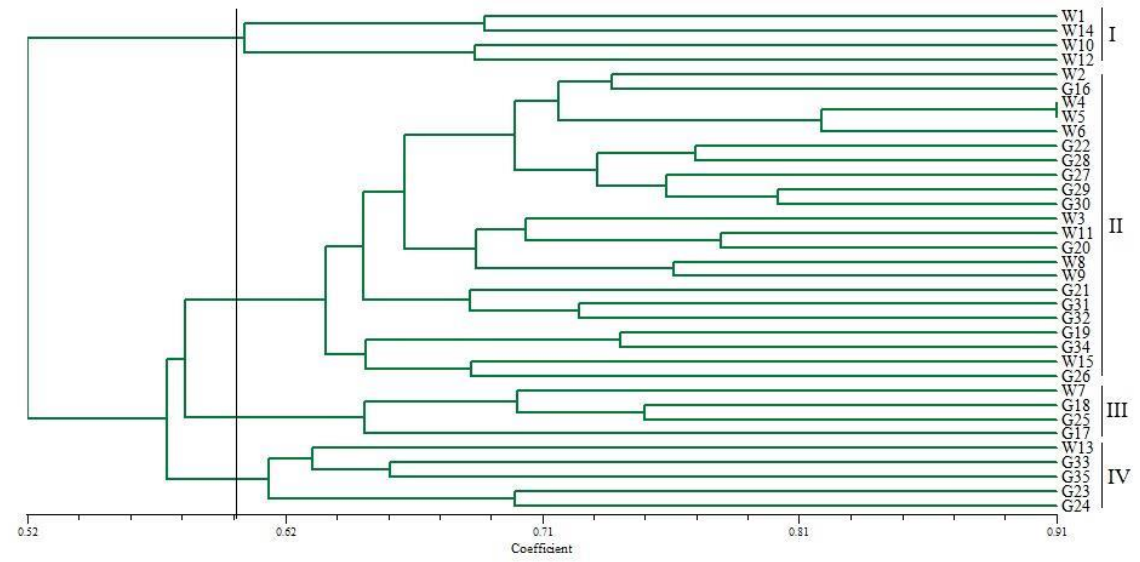

Fig. 2. UPGMA dendrogram of ISSR, IRAP, and REMAP analyses of 35 pistachio genotypes of Pistacia vera based on Jaccard's coefficient

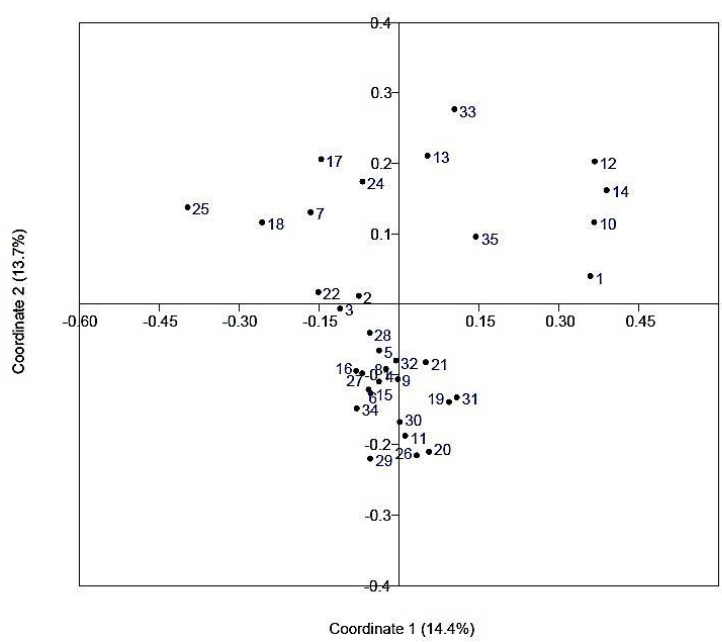

Fig. 3. Principal coordinate analysis (PCoA) showing Jaccard's genetic similarity among 35 pistachio genotypes of Pistacia vera. Accessions codes are identified in Table 1

Table 4. Levels of polymorphism and comparison of informativeness obtained with ISSR, IRAP, and REMAP markers in wild and cultivated pistachio genotypes

\begin{tabular}{|c|c|c|c|c|c|c|c|c|c|c|}
\hline Accession & Marker type & TB & $\mathrm{PB}$ & PPB & PIC & EMR & MI & $\mathrm{Ne}$ & $\mathrm{H}$ & I \\
\hline \multirow{4}{*}{$\begin{array}{l}35 \\
\text { pistachio } \\
\text { genotypes }\end{array}$} & ISSR & 8.60 & 7.00 & 82.83 & 0.30 & 5.73 & 1.78 & 1.63 & 0.34 & 0.49 \\
\hline & IRAP & 9.60 & 8.40 & 82.35 & 0.31 & 8.74 & 3.18 & 1.67 & 0.36 & 0.52 \\
\hline & REMAP & 8.00 & 7.20 & 88.69 & 0.32 & 6.55 & 2.26 & 1.68 & 0.37 & 0.53 \\
\hline & Mean & 8.01 & 7.00 & 84.62 & 0.31 & 6.07 & 2.02 & 1.66 & 0.36 & 0.52 \\
\hline \multirow{4}{*}{$\begin{array}{l}15 \\
\text { wild } \\
\text { genotypes }\end{array}$} & ISSR & 8.33 & 6.50 & 78.43 & 0.28 & 5.10 & 1.43 & 1.54 & 0.30 & 0.43 \\
\hline & IRAP & 8.14 & 7.14 & 85.20 & 0.33 & 6.35 & 2.31 & 1.61 & 0.34 & 0.49 \\
\hline & REMAP & 8.00 & 6.60 & 80.25 & 0.27 & 5.61 & 1.78 & 1.61 & 0.33 & 0.48 \\
\hline & Mean & 8.15 & 6.74 & 81.30 & 0.30 & 5.68 & 1.84 & 1.60 & 0.33 & 0.48 \\
\hline \multirow{4}{*}{$\begin{array}{l}20 \\
\text { cultivated } \\
\text { genotypes }\end{array}$} & ISSR & 8.33 & 6.33 & 75.15 & 0.28 & 4.91 & 1.45 & 1.56 & 0.33 & 0.46 \\
\hline & IRAP & 8.14 & 7.00 & 82.35 & 0.30 & 6.09 & 2.02 & 1.63 & 0.34 & 0.49 \\
\hline & REMAP & 8.00 & 6.80 & 84.69 & 0.30 & 5.83 & 1.82 & 1.61 & 0.33 & 0.48 \\
\hline & Mean & 8.15 & 6.71 & 80.73 & 0.29 & 5.61 & 1.77 & 1.62 & 0.33 & 0.49 \\
\hline
\end{tabular}

Note: see Table 3 
The mean of PIC value for all the primers used for the wild and cultivated genotypes were 0.30 and 0.29 , respectively (Table 4 ). In wild genotypes, the total average values of EMR, MI, $\mathrm{Ne}, \mathrm{H}$, and I for all the primers were $5.68,1.84,1.60,0.33$, and 0.48 , respectively; whereas for the cultivated genotypes, these values were $5.61,1.77,1.62,0.33$, and 0.49 , respectively (Table 4).

\section{Genetic relationships and cluster analysis}

The dendrogram of the 35 pistachio genotypes based on the ISSR, IRAP, and REMAP markers data was constructed based on Jaccard's similarity coefficient using the UPGMA method (Fig. 2). The results of the dendrogram showed that the 35 pistachio genotypes could be completely divided into four main groups based on the values of the similarity coefficient from 0.40 to 0.91 . The cophenetic correlation coefficient was calculated to evaluate the usefulness of the UPGMA method in clustering plant accessions. The high cophenetic correlation coefficient $(r=0.82)$ suggests that this dendrogram is a good representation of our ISSR, IRAP, and REMAP data. Genetic relationships among pistachio genotypes were also investigated by PCoA. The data generated from ISSR, IRAP, and REMAP markers were subjected to PCoA performed based on Jaccard's coefficient of similarity. In the PCoA analysis, the first two principal axes explain a total of $28.10 \%$ of genetic variation, with $14.40 \%$ and $13.70 \%$ for coordinates 1 and 2 , respectively (Fig. 3). The first two principal axes showed separation of the cluster IV, which confirmed the results of UPGMA clustering. A PCoA plot of the accessions is shown in Figure 3.

\section{DISCUSSION}

Several powerful molecular markers are currently available for genetic analysis of plant species. The choice of the most appropriate method is influenced by the purpose of the investigation and the biology and genetic structure of the species. Hence, the study of different techniques is needed to find the most appropriate technique for the issue being examined (Scariot et al. 2007). In the present study, three marker techniques, ISSR, IRAP, and REMAP, were used to assess the genetic relationships among wild-type genotypes and cultivated $P$. vera from Iran. To determine their utility in the discriminating and establishing genetic relationships in different pistachio genotypes, several approaches were followed. The level of polymorphism and a comparison of discriminating capacity of ISSR, IRAP, and REMAP markers are summarized in Table 3. Analysis of the amplification patterns of accessions belonging to the studied genotypes of $P$. vera showed a difference in position and number of generated bands. High level of polymorphism ratio was observed in all tested molecular marker systems. Ahmad et al. (2003) studied commercially important pistachio cultivars and recorded 46 alleles from 14 primer pairs, with an average of 3.3 loci per primer pair. The RAPD technique was applied to characterize Turkmenistan wild pistachio accessions by Barazani et al. (2003). They recorded 27 polymorphic bands of the total 62 RAPD fragments scored, with an average of 3.4 polymorphic bands per primer. In this study, using 18 ISSR, IRAP, and REMAP primer, we observed a total of 147 bands with an average of 7 polymorphic bands per primer. This indicates that primers used in this study, on an average, detected two times more polymorphism per primer than RAPDs or SSRs. On comparing the three techniques, the highest average number of polymorphic band per assay unit was amplified by REMAP technique (Table 3). Our investigation confirms that there is genetic variability among our research genotypes of $P$. vera and these markers with sufficient polymorphism could be used to investigate the genetic relationships among wild-type genotypes of $P$. vera. Shahi-Gharahlar et al. (2011) reported that wild and cultivated genotypes are rich resources of genes for breeding programs. The results are in agreement with the reports published earlier (Ghaemmaghami et al. 2013; Kırdök \& Çiftçi 2016). According to Xie et al. (2010), high, medium, and low loci polymorphism is appointed as PIC $>0.5 ; 0.5>\mathrm{PIC}>0.25$; and $\mathrm{PIC}<0.25$, respectively. The PIC value used for the assessment of genetic relationships in this experiment was 0.31 . The average value of $\mathrm{Ne}, \mathrm{H}, \mathrm{I}, \mathrm{EMR}$, and $\mathrm{MI}$ indicated that the background genetic data of germplasm accessions should be considered for integrated application in the breeding programs for germplasm improvement. 
It is necessary to mention that, as an assessment of genetic relationships, the $\mathrm{H}, \mathrm{I}$, and Ne were more appropriated than the actual number of alleles, because the latter depends on the sample size (Tajima et al. 1994). In the present study, the mean values of $\mathrm{Ne}, \mathrm{H}$, and I were $1.66,0.36$, and 0.52 , respectively. Previous researches have shown that $\mathrm{Ne}, \mathrm{H}$, and I values were 1.37, 0.23, and 0.37 using the SRAP marker in Punica granatum L. (Soleimani et al. 2012). The results suggest that REMAP and IRAP would be more useful than ISSR to investigate genetic relationships among pistachios genotypes. Comparison of diversity indices $\mathrm{Ne}, \mathrm{H}$, and I in commercial and wild pistachio genotypes indicates a high level of genetic diversity in commercial cultivars than wild genotypes in the studied area (Table 4). This might be due to the non-Iranian origin of the genotypes (Italiaei riz, Italiaei Dorosht and Kerman) or due to the local adaptation of genotypes that obtained from diverse geographical areas. Dendrograms based on UPGMA clustering of Jaccard's coefficient similarity were constructed with a cophenetic correlation coefficient of 0.82 using the Mantel test. Mohammadi and Prasanna (2003) reported that if the cophenetic correlation ranges between 0.8 and 0.9 , the dendrogram represents the similarity index strongly. Therefore, the cophenetic correlation coefficient supports the validity of the dendrogram. The general UPGMA dendrogram was constructed using the combined data of the three sets of molecular markers. The range of genetic similarity was from 0.40 to 0.91 . The tested genotypes were classified into four main clusters. There were high genetic relationships among pistachio cultivars. The presence of most cultivated genotypes in a group may suggest the existence of a limited genetic diversity among commercial pistachio cultivars as described previously (Arabnezhad et al. 2011; Shanjani et al. 2009). According to Shanjani et al. (2009), changing livelihood conditions may have had an effect in the reduction of genetic variation of pistachio; for example, in the past, the exchange of local cultivars among farmers was popular. This fact confirms our speculation that the cultivation of pistachio has increasingly led to the reduction of their genetic variation because of the usage of improved varieties and to the accessibility of private or public-grafted seedling nurseries for pistachio.
These results suggest that wild-type genotypes of $P$. vera are located in different clusters, which indicates its highly diverse genotypes, so that four genotypes W1, W10, W10, and W14 distinguished from the rest of $P$. vera genotypes, hence, could be a useful source of genetic material for a breeding program of pistachios in the future. Clustering of some of the wild-type genotypes along with cultivated pistachios in a group indicated that there are certain common alleles between them. The results demonstrate that low level of divergence among wild and cultivated $P$. vera promoting the hypothesis that the cultivated $P$. vera have been developed from wild accessions. This conclusion is similar to those suggested that pistachio cultivars are recently evolved and diverged from their "wild" relatives (Arabnezhad et al. 2011; Pazouki et al. 2010).

\section{CONCLUSION}

The results stated showed that ISSR, IRAP, and REMAP markers can reveal variability between pistachio genotypes. REMAP and IRAP assays are more reliable than ISSR because of their reproducible bands. The 5'LTR2 and 5'LTR2 + UBC811 primers with the highest PIC, the 5'LTR2 and Sukkula + UBC855 primers with the highest EMR and MI, and the Sukkula primer with the highest $\mathrm{Ne}$ and $\mathrm{H}$ were the most informative primers that could be used to assess the relationships of pistachio accessions. These results suggest that the wild pistachios available in natural habitat in northeastern of Iran represent a unique genetic resource that needs to be conserved and maintained. This can help in the provided diverse base of genetic material for developing new varieties and decrease the risk of the pistachio narrow genetic base.

\section{Acknowledgment}

This work was supported by University of Guilan. Authors would like to thank the research section of the University of Guilan for their help.

\section{REFERENCES}

Ahmad R., Ferguson L., Southwick S.M. 2003. Identification of pistachio (Pistacia vera $\mathrm{L}$.) nuts with microsatellite markers. Journal of the American Society for Horticultural Science 128(6): 898-903. DOI: 10.21273/jashs.128.6.0898. 
Arabnezhad H., Bahar M., Tajabadi Pour A. 2011. Evaluation of genetic relationships among Iranian pistachios using microsatellite markers developed from Pistacia khinjuk Stocks. Scientia Horticulturae 128(3): 249 254. DOI: 10.1016/j.scienta.2011.01.028.

Barazani O., Atayev A., Yakubov B., Kostiukovsky V., Popov K., Golan-Goldhirsh A. 2003. Genetic variability in Turkmen populations of Pistacia vera L. Genetic Resources and Crop Evolution 50(4): 383 389. DOI: $10.1023 / \mathrm{a}: 1023928017410$.

Crane J.C. 1979. Pistachio nuts. In: Woodroof J.G. (Ed.), Tree nuts: production, processing, products, $2^{\text {nd }}$ ed. USA, AVI Publishing, $731 \mathrm{p}$.

Dempewolf H., Baute G., Anderson J., Kilian B., Smith C., Guarino L. 2017. Past and future use of wild relatives in crop breeding. Crop Science 57(3): 1070 1082. DOI: 10.2135/cropsci2016.10.0885.

Ghaemmaghami L., Attar F., Rahiminejad M.R. 2013. Distinctness and inter relationships of Pistacia L. species in Iran as evidenced by retroelement insertional polymorphisms (IRAP method). Iranian Journal of Botany 19(1): 78-85. DOI: 10.22092/ijb.2013.2998.

He S.-A., Yi T.-S., Pei S.-J., Huang H.-W. 2015. Crop plants and their wild relatives. In: Hong D.-Y., Blackmore S. (Eds.), Plants of China: A companion to the flora of China. Cambridge University Press, UK, p. 283-308.

Hormaza J.I., Pinney K., Polito V.S. 1998. Genetic diversity of pistachio (Pistacia vera, Anacardiaceae) germplasm based on randomly amplified polymorphic DNA (RAPD) markers. Economic Botany 52(1): 78-87. DOI: $10.1007 / \mathrm{bf02861298.}$

Kalendar R., Flavell A.J., Ellis T., Sjakste T.H.N., Moisy C., Schulman A.H. 2011. Analysis of plant diversity with retrotransposon-based molecular markers. Heredity 106(4): 520-530. DOI: 10.1038/hdy.2010.93.

Kalendar R., Grob T., Regina M., Suoniemi A., Schulman A. 1999. IRAP and REMAP: two new retrotransposon-based DNA fingerprinting techniques. Theoretical and Applied Genetics 98(5): 704-711. DOI: 10.1007/s001220051124.

Kar P.K., Vijayan K., Mohandas T.P., Nair C.V., Saratchandra B., Thangavelu K. 2005. Genetic variability and genetic structure of wild and semi-domestic populations of tasar silkworm (Antheraea mylitta) ecorace Daba as revealed through ISSR markers. Genetica 125(2-3): 173-183. DOI: 10.1007/s10709-005-7002-z.

Kırdök E., Çiftçi Y.Ö. 2016. Retrotransposon marker systems as a tool to analyze molecular diversity of
Mediterranean Pistacia species. International Journal of Agriculture and Biology 18(3): 601-606. DOI: $10.17957 / \mathrm{ijab} / 15.0132$.

Michel D. 2017. Iran's Impending Water Crisis. In: Reed D. (Ed.), Water, Security and U.S. Foreign Policy. Routledge, USA, pp. 168-188. DOI: 10.4324/9781315168272-10.

Mohammadi S.A., Prasanna B.M. 2003. Analysis of genetic diversity in crop plants - salient statistical tools and considerations. Crop Science 43(4): 1235-1248. DOI: 10.2135/cropsci2003.1235.

Parfitt D.E., Badenes M.L. 1997. Phylogeny of the genus Pistacia as determined from analysis of the chloroplast genome. Proceedings of the National Academy of Sciences of the USA 94(15): 7987-7992. DOI: 10.1073/pnas.94.15.7987.

Pazouki L., Mardi M., Shanjani P.S., Hagidimitriou M., Pirseyedi S.M., Naghavi M.R. et al. 2010. Genetic diversity and relationships among Pistacia species and cultivars. Conservation Genetics 11(1): 311318. DOI: 10.1007/s10592-009-9812-5.

Pell S.K. 2004. Molecular systematics of the cashew family (Anacardiaceae). LSU Doctoral Dissertations, 193 p.

Powell W., Morgante M., Andre C., Hanafey M., Vogel J., Tingey S., Rafalski A. 1996. The comparison of RFLP, RAPD, AFLP and SSR (microsatellite) markers for germplasm analysis. Molecular Breeding 2(3): 225-238. DOI: 10.1007/bf00564200.

Reddy M.P., Sarla N., Siddiq E.A. 2002. Inter simple sequence repeat (ISSR) polymorphism and its application in plant breeding. Euphytica 128(1): 9-17. DOI: 10.1023/a:1020691618797.

Rohlf F. 2009. NTSYSpc. Numerical taxonomy and multivariate analysis system, version 2.2. Exeter Software, Applied Biostatistics, USA.

Scariot V., De Keyser E., Handa T., De Riek J. 2007. Comparative study of the discriminating capacity and effectiveness of AFLP, STMS and EST markers in assessing genetic relationships among evergreen azaleas. Plant Breeding 126(2): 207-212. DOI: 10.1111/j.1439-0523.2007.01326.x.

Shahi-Gharahlar A., Zamani Z., Fatahi R., Bouzari N. 2011. Estimation of genetic diversity in some Iranian wild Prunus subgenus Cerasus accessions using inter-simple sequence repeat (ISSR) markers. Biochemical Systematics and Ecology 39(4): 826833. DOI: 10.1016/j.bse.2011.07.018.

Shanjani P.S., Mardi M., Pazouki L., Hagidimitriou M., Avanzato D., Pirseyedi S.M. et al. 2009. Analysis of the molecular variation between and within cultivated and wild Pistacia species using AFLPs. 
Tree Genetics and Genomes 5(3): 447-458. DOI: 10.1007/s11295-008-0198-1.

Smith J.S.C., Chin E.C.L., Shu H., Smith O.S., Wall S.J., Senior M.L. et al. 1997. An evaluation of the utility of SSR loci as molecular markers in maize (Zea mays L.): comparisons with data from RFLPS and pedigree. Theoretical and Applied Genetics 95(1): 163-173. DOI: 10.1007/s001220050544.

Soleimani M.H., Talebi M., Sayed-Tabatabaei B.E. 2012. Use of SRAP markers to assess genetic diversity and population structure of wild, cultivated, and ornamental pomegranates (Punica granatum L.) in different regions of Iran. Plant Systematics and Evolution 298(6): 1141-1149. DOI: 10.1007/s00606-012-0626-4.

Sorkheh K., Amirbakhtiar N., Ercisli S. 2017. Retraction note to: Potential start codon targeted (SCoT) and interretrotransposon amplified polymorphism (IRAP) markers for evaluation of genetic diversity and conservation of wild Pistacia species population. Biochemical Genetics 55(5): 421-422. DOI: 10.1007/s10528-017-9830-9.

Tajima F., Tokunaga T., Miyashita N.T. 1994. Statistical methods for estimating the effective number of alleles, expected heterozygosity and genetic distance in self-incompatibility locus. Japanese Journal of Genetics 69(3): 287-295. DOI: 10.1266/jjg.69.287.

Xie W.-G., Zhang X.-Q., Cai H.-W., Liu W., Peng Y. 2010. Genetic diversity analysis and transferability of cereal EST-SSR markers to orchardgrass (Dactylis glomerata L.). Biochemical Systematics and Ecology 38(4): 740-749. DOI: 10.1016/j.bse.2010.06.009.

Yang H.-B., Kang W.-H., Nahm S.-H., Kang B.-C. 2015. Methods for developing molecular markers. In: Koh H.-J., Kwon S.-Y., Thomson M. (Eds.), Current Technologies in Plant Molecular Breeding. Springer, p. 15-50. DOI: 10.1007/978-94-017-9996-6_2.

Yeh F.C., Boyle T., Rongcai Y., Ye Z., Xiyan J.M. 1999. POPGENE version 1.31: Microsoft Window-based Freeware for Population Genetic Analysis. Quick User Guide, 28 p.

Ziya Motalebipour E., Kafkas S., Khodaeiaminjan M., Çoban N., Gözel H. 2016. Genome survey of pistachio (Pistacia vera L.) by next generation sequencing: Development of novel SSR markers and genetic diversity in Pistacia species. BMC Genomics 17; 998; 14 p. DOI: 10.1186/s12864-016-3359-x.

Zohary M. 1952. A monographical study of the genus Pistacia. Palestine Journal of Botany (Jerusalem Series) 5(4): 187-228. 